ESTUDIO ESPECIAL

\title{
ENCUESTA DE PORTADORES DE NEISSERIA MENINGITIDIS EN EL ÁREA DE SALUD DE GRAN CANARIA (*)
}

\author{
Amós García Rojas (1), Ana Bordes Benítez (2), Bernardo Lafarga Capuz (2), Julio Vázquez Moreno (3), \\ Elena López Villarrubia (1), Pilar García Castellano (1) y José Solís Romero (1).
}

(1) Sección de Promoción de la Salud. Dirección General de Salud Pública.

(2) Laboratorio de Microbiología. Hospital Nuestra Señora del Pino. Gran Canaria.

(3) Laboratorio de Meningococos. Centro Nacional de Microbiología. Instituto de Salud Carlos III.

$\left(^{*}\right)$ Estudio becado por el Ilustre Colegio Oficial de Médicos de Las Palmas.

\section{RESUMEN}

Fundamentos: Se plantea A) Conocer la tasa de portadores y los tipos circulantes de Neisseria Meningitidis en la población residente en el área de salud de Gran Canaria. B) Conocer el patrón de distribución de estos portadores.

Métodos: Se realizó un diseño descriptivo transversal, con un muestreo aleatorio en etapas múltiples y por conglomerados. Se detenninó un tamaño muestral mínimo de 707 personas para una prevalencia esperada de $18,6 \%$, con una confianza del $95,6 \%$ y precisión de 0,02 . A sumiendo que un $15 \%$ de las personas no quisieran colaborar, se incrementó el tamaño muestral a 831 personas, distribuidas en cada conglomerado de manera proporcional a la población existente. Este tamaño se distribuyó a su vez, en cuatro grandes grupos de edad y sexo, proporcionalnente a su importancia en cada zona básica de salud seleccionada aleatoriamente. Los individuos de la muestra se identificaban entre los que actulian a las unidades de extracción, y una vez superados los criterios de exclusión se les solicitaba su colaboración woluntaria en el estudio. Si aceptaban, se les cumplimentaba un :Lestionario que englobaba diferentes variables de interés epidemiológico y se les realizaba un frotis faríngeo. Al haber seleccionado los equipos de Atención Primaria con muestreo aleatorio simqle y seguir el misno método para elegir los individuos dentro de ellos, la estimación de la prevalencia se realizó mediante estimador no sesgado.

Resultados: .Se obtuvieron un total de 828 muestras, lo que supuso un $99,6 \%$ de las previstas. Salvo tres, todos los individuos seleccionados participaron voluntariamente en el estudio, lo que le confiere una alta representatividad. Toclas las cepas obtenidas correspondian a N. Meningitidis Serogrupo B, salvo una identificada como N. Meningitidis Serognupo C Sero/Subtipo 4:P1 2,5. Las cepas de N. Meningitidis serogrupo B identificadas, correspondian a 25 serosubtipos diferentes. La prevalencia puntual tras haber estudiado la muestra fue de $6,45 \%$, la varianza $=0,0275$ y el enror estándar $=1,66$. Podemos afirmar con una confianza del $95 \%$, que la prevalencia de portadores de N. Meningitidis en el área de salud de Gran Canaria, se estima entre el $3,2 \%$ y el $9,7 \%$.

Conclusiones: Se comprueba un claro predorninio de cepas N. Meningitidis serogrupo B entre los portadores. No aparecen diferencias estadísticamente significativas en la prevalencia observada entre los distintos grupos de edad, ui entre ambos sexos.
ABSTRACT

\section{Survey of $N$. Meningitidis Carriers in the Gran Canary Health Jurisdiction}

Background: A) To ascertain the rate of carriers and the Types of Neisseria Meningitidis circulating in the population resident in the health jurisdiction of Gran Canaria. B) to ascertain the pattern of discribution of such carriers.

Methods: A descriptive transversal design was made, with a random sampling in multiple stages and by conglomerates. A minimum sample size was deternined at 707 individuals for an expected prevalence of $8.6 \%$, with a rate of reliability of $95.6 \%$ and a precision of 0.02 . Assuming that $15 \%$ if the individuals would not be willing to co-operate, the sample size was increased to 831 individuals, distributed in each conglomerate in proportion to the existing population. This size was distributed in tum into four groups by age and sex, in nqroportion to their significance in each basic health care zone selected at random. The individuals in the sample were identified from among those who attended the blood extraction units, and after they had passed the criteria of exclusion, their co-operation was requested as volunteers in the study. If they accepted, a questionnaire was filled out with a number of variables of epidemiological interest and a pharyngeal smear was taken. Since the Primary Care units were selected on a simple random basis, and the sarne method was used to select the individuals willin the units, the estinlate of the prevalence was made by meins of an unbiased estimator.

Results: A total of 828 samples were obtained, that is, $99.6 \%$ of the number foreseen. With the exception of three, all of the individuals selected participated voluntarily in the study, a circunstance rendering it highly representative. All of the strains obtained corresponded to N. Meningitidis Serogroup B, except for one identified as N. Meningitidis Serogroup C Sero/Subtype 4:Pl 2,5. The strains of N. Meningitidis serogroup B identified corresponded to 25 different sero-subtypes. The prevalence deternined after having studied the sample was $6,45 \%$, the variance $=0.0275$ and the standard error $=1.66$. We can conclude with a $95 \%$ degree of reliability that the prevalence of carriers of $\mathrm{N}$. Meningitidis in the Gran Canaria health jurisdiction is estimated to be between $3.2 \%$ and $9.7 \%$.

Conclusions: A clear predominance of N. Meningitidis serogroup $B$ strains among carriers has been verified. There are no statistically significant differences in the prevalence abserved among the different age gmups nor between sexes. 


\section{INTRODUCCIÓN}

El comportamiento epidemiológico de la enfermedad meningocócica en Canarias ha sido muy similar al verificado en el resto del país, con ciclos multianuales, un último gran pico epidémico en el año 1979, un posterior descenso hasta comienzos de la década de los 90 , y una incidencia que a diferencia del resto del Estado, no presenta carácter estacional apareciendo casos a lo largo de todo el aก̃o.

La presentación de la enfermedad ha estado ligada a un predominio del serogrupo B, hasta que a mitad de la década de los 90 se observó una tendencia creciente de las cepas de serogrupo $C$, verificándose en la temporada 1996-1997 un predominio de estas cepas, fundamentalmente de la identificada como $\mathrm{C}: 2 \mathrm{~b}: \mathrm{P} 1.2,5$. Este proceso se apreció más claramente en el área de salud de Gran Canaria (en Canarias, según la Ley de Ordenación Sanitaria, cada isla es un área de salud).

En el resto del Estado, esta tendencia creciente se había detectado a partir de la temporada 1991-1992, en los aislamientos efectuados en algunas zonas del oeste del país. En la temporada 1995-1996 se produjo un predominio generalizado del serogrupo $\mathrm{C}$ y tras el inicio de la temporada 1996-1997, se comprobó un aumento en el número de casos de enfermedad meningocócica declarados semanalmente. Sin embargo, la incidencia en nuestra Comunidad Autónoma permaneció baja.

La experiencia acumulada en otros países en el control de situaciones semejantes, llevó a que todas las Comunidades Autónomas desarrollaran intervenciones de vacunación, salvo Navarra y Canarias.

En nuestra Comunidad Autónoma, la nueva situación epidemiológica generada por esta enfermedad caracterizada como hemos visto, por un cambio cn el serogrupo prevalente y una incidencia baja, condujo a una estrategia de intervención basada en:
- La actuación enérgica ante casos y brotes epidémicos, con quimioprofilaxis y vacunación a contactos, según se define en el protocolo de la Red Nacional de Vigilancia Epidemiológica y

- Favorecer distintas líneas de investigación aplicadas al conocimiento epidemiológico de la enfermedad meningocócica.

De esta manera, desde el Servicio de Epidemiología en la provincia de Las Palmas, se planteó realizar el presente estudio.

Objetivos:

A) Conocer la tasa de portadores y los tipos circulantes de Neisseria Meningitidis en la población residente en el área de salud de Gran Canaria.

B) Conocer el patrón de distribución de portadores de Neisseria Meningitidis en la población residente en el área de Gran Canaria.

\section{MATERIAL Y MÉTODOS}

\section{Diseño de la muestra}

Se realizó un diseño descriptivo transversal, con un muestreo aleatorio en etapas múltiples y por conglomerados. Teniendo en cuenta que el $98 \%$ de la población de la isla está adscrita al Servicio Canario de Salud, en una primera etapa se hizo un muestreo por conglomerados utilizando como unidad primaria la zona básica de salud. De las 51 zonas básicas de salud del Area, se eligieron aleatoriamente 11 para participar en el estudio: Agüimes (16.156 habitantes), Santa María de Guía (10.937), Vecindario (36.210), Vega de San Mateo (6.153), Isleta (24.548), Alcaravaneras (26.456), Miller Bajo (34.265), Guanarteme (35.145), Barrio Atlántico (15.805), San Gregorio (25.264) y El Calero (16.578).

Para la selección de la muestra final, se realizó en una segunda etapa, un muestreo 
aleatorio simple en cada una de las zonas básicas de salud, teniendo en cuenta que se conocía exactamente la población que depende de cada Centro.

Se determinó un tamaño muestral mínimo de 707 personas para una prevalencia esperada del $8,6 \%$, con una confianza del $95,6 \%$ y precisión de 0,02. Asumiendo que un $15 \%$ de las personas no quisieran colaborar, se incrementó el tamaño muestral a 831 personas, distribuidas en cadå conglomerado proporcionalmente a la población existente. Este tamaño se distribuyó a su vez, en cuatro grandes grupos de edad y sexo, proporcionalmente a su importancia en cada zona básica de salud seleccionada aleatoriamente, según el Censo de Población y Vivienda del Instituto Canario de Estadística.
Los individuos de la muestra se identificaron entre los que acudían a las unidades de extracción. Allí, se les solicitaba su colaboración voluntaria en el estudio y si aceptaban, se les cumplimentaba un cucstionario que englobaba diferentes variables de interés epidemiológico y se les realizaba un frotis faríngeo.

Al haber seleccionado los equipos de Atención Primaria con muestreo aleatorio simple y seguir el mismo método para elegir dentro de ellos a los individuos, la estimación de la prevalencia se realizó mediante el estimador no sesgado:

$$
\mathrm{P}=\frac{\mathrm{M}}{\mathrm{N}_{\mathrm{m}}} \sum_{\mathrm{i}=1}^{\mathrm{n}} \mathrm{N}_{\mathrm{i}} \mathrm{p}_{\mathrm{i}}
$$

y la varianza:

$$
\operatorname{Var}(P)=\frac{1}{N_{2}}\left[\frac{m^{2}(M-m) m}{M_{n}(m-1)}\right] \sum_{i=1}^{m}\left(n_{i} p_{i}-\frac{1}{m} \sum_{i=1}^{m} N_{i} p_{i}\right)^{2}+\frac{M}{m} \sum_{i=1}^{m} \frac{N_{i}-n_{i}}{n_{i}} \cdot \frac{p_{i} \cdot q_{i}}{n_{i}-1}
$$

donde: salud.

$\mathrm{N}_{\mathrm{i}}=\mathrm{N}^{\mathrm{o}}$ de individuos de los centros de

$\mathrm{n}_{\mathrm{i}}=\mathrm{N}^{\mathrm{o}}$ de individuos de los centros de salud seleccionados.

$\mathrm{M}=\mathrm{N}^{\mathrm{o}}$ de centros de salud.

$\mathrm{m}=\mathrm{N}^{\circ}$ de centros de salud seleccionados.

$$
\mathrm{N} \rightarrow \sum_{\mathrm{i}=1}^{\mathrm{M}} \mathrm{N}_{\mathrm{i}}=\mathrm{N}^{\mathrm{o}} \text { total de individuos en }
$$

los centros de salud.

$$
n \rightarrow \sum_{i=1}^{m} n_{i}=N^{o} \text { total de individuos en }
$$

los centros de salud seleccionados.

\section{Toma y análisis de las muestras}

Con anterioridad a la toma de muestras, se realizaron reuniones informativas con los coordinadores/as médicos/as y responsables de enfermería de las zonas básicas de salud seleccionadas aleatoriamente.

La toma de muestras tuvo lugar en dos etapas, la primera de mayo a julio de 1999 y la segunda de septiembre a octubre de ese mismo año. Fue efectuada por personal del servicio de epidemiología en la provincia de Las Palmas adiestrado para tal fin.

Los criterios de exclusión para participar en el estudio fueron: Individuos

- Que no residieran habitualmente en Gran Canaria.

- Que presentaran patologías o estuvieran sometidos a tratamiento, especialmente quimioterápico/antibiótico.

- Que hubieran sido vacunados frente a la enfermedad meningocócica $\mathrm{A}+$ C.

- Que presentaran alguna condición que se estimara pudiera alterar los resultados. 
Las muestras se enviaban el mismo día de realización del frotis faríngeo y en período de tiempo inferior a tres horas, al Laboratorio de Microbiología del Hospital Nuestra Señora del Pino, en torunda estéril con medio de transporte. En el laboratorio se procedía a la siembra en medio selectivo Tayer-Martín. Si se producía crecimiento se verificaba la confirmación de diplococos gram negativos, oxidasa positivos, bioquímicamente meningococos. En este caso, se procedía mediante técnicas de serogrupaje a identificación de serogrupo. Los serogrupos obtenidos se enviaban en placa agar-chocolate, y el mismo día de su obtención, al Laboratorio de Referencia de Meningococos del Centro Nacional de Microbiología del Instituto de Salud Carlos III, donde se procedía a su subtipaje.

\section{RESULTADOS}

Se obtuvieron un total de 828 muestras, lo que supuso un $99,6 \%$ de las previstas. Salvo tres, todos los individuos seleccionados participaron voluntariamente en el estudio, lo que le confiere una alta representatividad. La muestra estuvo constituida por un $50,06 \%$ de mujeres y un $49,94 \%$ de hombres, distribuidos/as según los siguientes estratos de edad:
La distribución en la población de $\mathrm{N}$. Lactámica y N. Meningitidis seguía el patrón habitual, con una mayor tasa de portadores de N. Lactámica en los niños/as más pequeños, tasa que iba disminuyendo con la edad.

Todas las cepas obtenidas correspondían a N. Meningitidis Serogrupo B, salvo una, identificada como N. Meningitidis Serogrupo C Sero/Subtipo 4:P1.2,5. Las cepas de N. Meningitidis serogrupo B identificadas, correspondían a 25 tipos diferentes.

Tras haber estudiado la muestra, la prevalencia puntual fue de $6,45 \%$, la varianza $=$ 0,0275 y el error estándar $=1,66$.

Podemos afirmar con una confianza del $95 \%$, que la prevalencia de portadores de $\mathrm{N}$. Meningitidis en el área de salud de $\mathrm{Gran} \mathrm{Ca}$ naria, se estima entre el $3,2 \%$ y el $9,7 \%$.

Teniendo en cuenta que la prevalencia puntual obtenida fue del $6,45 \%$, se estimó la que existía en los diferentes grupos de edad de la muestra, recalculando el error de la estimación:

A su vez, se siguió el mismo proceso para ver si en la muestra existían diferencias entre ambos sexos:

Tabla 1

Distribución de la muestra según grupos de edad y sexo

\begin{tabular}{|lccc|}
\hline & & Sexo & \\
Grupos de Edad & Hombres & Mujeres & \\
& & & Total \\
\cline { 2 - 4 } & 140 & 133 & 273 \\
\hline 0 a 19 años & 168 & 163 & 331 \\
20 a 44 años & 68 & 79 & 157 \\
45 a 64 años & 29 & 41 & 70 \\
\hline 65 y más años & 415 & 416 & 831 \\
\hline Total & & & \\
\hline
\end{tabular}


Tabla 2

Prevalencia de $\boldsymbol{N}$. Meningitidis en los distintos grupos de edad de la muestra

\begin{tabular}{|lcc|}
\hline Grupos de edad & Prevalencia & Intervalo de confianza \\
\hline 0 a 19 años & 3,66 & $0,7-6,5 \%$ \\
20 a 44 años & 7,25 & $4,65-9,89 \%$ \\
45 a 64 años & 2,54 & $0-6,38 \%$ \\
\hline
\end{tabular}

Tabla 3

Prevalencia de $N$. meningitidis en la muestra según sexo

\begin{tabular}{|c|c|c|}
\hline Sexo & Prevalencia & Intervalo de confianza \\
\hline Hombre & $5 \%$ & $2,64-7,36 \%$ \\
\hline Mujer & $4 \%$ & $1,64-6,3 \%$ \\
\hline
\end{tabular}

\section{CONCLUSIONES}

- Se corrobora la distinta distribución en relación a la edad, que mantienen la N. Lactámica y la N. Meningitidis entre los portadores identificados.

- Se comprueba un claro predominio de cepas N. Meningitidis serogrupo $\mathrm{B}$, entre los portadores.

- No aparecen diferencias estadísticamente significativas en la prevalencia observada entre los distintos grupos de edad, ni entre ambos sexos.

\section{Limitaciones del estudio}

- Las derivadas del marco del muestreo: Había una pérdida de población que no utiliza la red sanitaria asistencial pública, y otra de población marginal.

- La muestras obtenidas correspondían a exudados faríngeos, ya que al intentar realizar frotis nasoforingeo, los individuos identificados y que voluntariamente querían participar en el estudio, rechazaban en su totalidad este mecanismo por las molestias que les producían.

\section{" BIBLIOGRAFÍA}

1. Boletín Oficial del Estado. Real Decreto 2210/1995 por el que se crea la red nacional de vigilancia epidemiológica. BOE núm. 21 , 24/01/1996.

2. Boletín Oficial de Canarias. Decreto $165 / 1998$ pro el que se crea la red canaria de vigilancia epidemiologica y se establecen las normas para regular su funcionamiento. BOC núm. 127, 7/10/1998.

3. Centro Nacional de Epidemiología. Protocolos de las enfermedades de declaración obligatoria. Madrid: Ministerio de Sanidad y Consumo; 1997.

4. Portadores de N. Meningitidis e Incidencia de Enfermedade Meningocócica. Bol Epidemiol Galicia 1997; 10(3):1-2.

5. Peralta Peralta I. «Encuesta de portadores y caracterización de cepas circulantion Neisseria 
Meningitidis en Extremadura». Rev Esp Salud Pública 1998; 72(5):451-453.

6. Vázquez JA, De la Fuente L, Berrón S. Infección meningocócica. Informe del laboratorio de referencia de meningococos sobre estado actual de serogrupos (enero-diciembre 1996). Bol Epidemiol Sem 1996; 4:205-212.

7. Mateo S, Cano R, García C. Chanching epidemiology of meningococcal disease in Spain, 1989-1997. Eurosurveillance 1997; 2:71-74.

8. Harrison LH. The worldwide prevention of meningococcal infection: still an elusive goal (editorial). JAMA 1995; 273:419-421.
9. Jackson LA, Schuchat A, Reeves MW, Wenger JD. Serogroup $C$ meningococcal oubreaks in the United States. JAMA 1995; 273:383-389.

10. De Wals P, Dionne M, Douville M, Boulianne N, Drapeau J, De Serres G. Impact of a mass inmunization campaing against serogroup $\mathrm{C}$ meningococcus in the province of Quebec, $\mathrm{Ca}$ nada. Bull World Health Organ 1996; 74:407. 411 .

11. Kriz P, Vickoka J, Bobak M. Targeted vaccination with meningococcal polysaccharide vaccine in one district of the Czech Republic. Epidemiol Infect 1995; 115:411-418. 